\title{
Impedance Control for Legged Robots: An Insight Into the Concepts Involved
}

\author{
Juan Carlos Arevalo and Elena Garcia
}

\begin{abstract}
The application of impedance control strategies to modern legged locomotion is analyzed, paying special attention to the concepts behind its implementation which is not straightforward. In order to implement a functional impedance controller for a walking mechanism, the concepts of contact, impact, friction, and impedance have to be merged together. $A$ literature review and a comprehensive analysis are presented compiling all these concepts along with a discussion on position-based versus forcebased impedance control approaches, and a theoretical model of a robotic leg in contact with its environment is introduced. A theoretical control scheme for the legs of a general legged robot is also introduced, and some simulations results are presented.
\end{abstract}

Index Terms-Contact, impedance control, legged robots.

\section{INTRODUCTION}

$\mathbf{R}$ OBOTICS research has been traditionally focused on industrial applications. However, with the evolution of technology, applications of robotics research have been broadened taking interest in applications not only to aid humans in repetitive tasks, but to aid them in their everyday lives too. One of these applications that have been growing over the past decade is assistive exoskeletons, which are essentially mobile robots wearable by a person enhancing his/her capabilities. Most complex work done in this field is focused on lower limb exoskeletons such as Berkeley Lower Extremity Exoskeleton (BLEEX) [1], Hybrid Assistive Limb-5 (HAL-5) [2], and the Nanyang Technological Universit (NTU) [3]. Moreover, there is also some work toward energy efficiency [4], where the possibility to use passive mechanisms in the structural design of exoskeletons is explored, and in the human-robot interaction, some exoskeletons use myographical signals to acquire the user's intention of motion [5], [6].

There is also an increasing interest in new applications of field robotics, especially in walking robots to carry loads on rough terrain, where wheeled vehicles cannot have access or simply it is not desirable to cause too much damage to the terrain.
For that purpose, research on agile quadrupeds is increasing. Probably, the most popular of these robots is BigDog [7] by Boston Dynamics; nonetheless, there are a few others, e.g., Kolt [8] at Stanford University, HyQ [9] at the Italian Institute of Technology, and HADE [10] at the Spanish National Research Council (CSIC) which are under development.

Nevertheless, research on industrial applications is far from abandoned; there is research going on in what is called intelligent assistive devices (IADs) [11] and on improving the performance of existing technology, i.e., application of genetic algorithms for task optimization [12].

Most of these applications require the robots to be in contact with the environment as well as with humans. Therefore, there is a need to control the contact force in order to prevent harming humans or damaging either the robot or the environment. This force can be controlled by means of direct force control; however, this approach has some issues. For example, using just force control it is not possible to reach a commanded position, for that reason, tasks that involve movement are not possible. One solution to that problem consists in decoupling the coordinates, where the robot is exerting force with the coordinates, where the robot is needed to move (hybrid position force control) [14] . Yet another problem is the fact that when the robot is in an unstructured environment (which is generally the case for walking robots), it is necessary to allow some deviation in both commanded force and position in order to prevent undesired events, such as damage to the robot or the environment or harm to humans. For that purpose, impedance control is a good option to control robots that involve contact, e.g., object manipulation and walking.

The objective of impedance control is not to directly control position or force, but the relationship between them. This allows reducing or increasing apparent stiffness, damping, or mass depending on the task. Moreover, with a single control scheme it is possible to control both position and force which is extremely useful in applications, where the robot is switching between constrained (in contact) and unconstrained motions (free motion).

Impedance control has been extensively studied, and it has been successfully implemented in the past. However, with the new course of robotics research new issues have arisen, and therefore, the requirements for impedance control strategies have changed. In order to understand how to adapt impedance control to these requirements, it is necessary to study the concepts that are involved in an impedance control strategy.

This study focuses on impedance control for legged locomotion since this application covers most of the problems for robot interaction. Most of the previous research on impedance 
control for legged locomotion is aimed at statically stable walking robots [15]-[17], and it focuses on the implementation of the control algorithm itself leaving aside the concepts behind the impedance controlled locomotion such as impact and friction. Legged locomotion has progressed to agile, dynamic walkers, where contact with the ground becomes a destabilizing issue, and it is the source of energy loss during locomotion [13]. Some modern research on the topic can be found in [18] and [19], where the problem of controller robustness against unknown perturbations (impact with unknown obstacles, etc.) is addressed. As legged robots are assumed to tackle terrains having different stiffness, the adjustment of the combined robot-ground impedance is of great importance to reduce the energy that is lost in the contact.

It is true that the added compliance could interfere the dynamic stability of the robot; however, there have been some approaches to combine the impedance control and the dynamic balance of the machine in a single control scheme. For that reason, the concepts of impact and friction need to be studied.

In spite of all the research effort on the subject, the application of impedance control is not straightforward. The implementation has to take into consideration all the aforementioned concepts. The aim of this study is to compile the basic necessary knowledge on these concepts since up to now it is scattered in many disciplines and, moreover, to provide an insight into them and a brief guide on the design of the proper impedance control approach for dynamic legged locomotion. To these authors' best knowledge, such compilation cannot be found in the current literature. In addition, the stability analysis that was performed by Lawrence [21] was extended to include the robot mass, and some simulation results are included using the leg model and the control algorithm that is proposed in this work.

This paper is organized in the following way: Section II provides a brief overview on the general impedance control strategy; Section III describes the two basic approaches in impedance control and states a practical comparison between them; Section IV reviews the contact models that are available in the literature and refers the reader to some relevant works on the subject; in Section $\mathrm{V}$ a model of a leg in contact with its environment is derived, and a brief discussion on friction modeling is done; Section VI discusses the application of impedance control to legged locomotion; Section VII presents simulation results; and finally in Section VIII the conclusion is stated.

\section{IMPEDANCE CONTROL}

Impedance Control is based on the following postulate: "It is impossible to devise a controller which will cause a physical system to present an apparent behavior to its environment which is distinguishable from that of a purely physical system" [22]. This postulate is a reasonable assumption, and it indicates that a controlled physical system can be described as an equivalent one.

A very practical and easy way to describe a system and its relationship to its environment is to use the interrelationship between its physical quantities (velocity, current, force, and voltage). Moreover, by inspecting the physical properties of such quantities, there is equivalence in the behavior of force and voltage, and velocity and current; therefore, it is possible to extend the concept, very well known in electrical systems, of impedance to mechanical systems. The mechanical impedance and admittance are, therefore, defined, respectively, by

$$
Z=\frac{F}{\dot{x}}, \quad Y=\frac{\dot{x}}{F} .
$$

The aforementioned equation is exchangeable in linear systems; however, in many important mechanical problems, such as manipulation and locomotion, the systems are nonlinear. Nonlinearity implies that the concepts of impedance and admittance are no longer exchangeable. The reason for this is that there exist physical systems such that can be described as an admittance, but not as an impedance and vice versa; for example, a solid ground that can always be pushed on, but cannot be moved, can only be described as an admittance. The author of [22] discussed this problem by giving some examples and stated that the most important consequence of the dynamic interaction between two physical systems is that they must complement each other, i.e., if one is an impedance, the other must be an admittance, and vice versa. It is obvious that a system can be controlled either by its admittance or by its impedance; nevertheless, according to the aforementioned restrictions one must choose whether to model it as an admittance or as an impedance considering its environment's physical characteristics.

In many robotic applications, the environment can be described as an admittance (e.g., manipulation and walking). Such is the case of the ground, since it can accept forces but it cannot be moved, as well as the case of kinematically constrained objects. Therefore, in order to ensure compatibility when the systems are coupled, the robot should behave as an impedance.

The authors of [23] defined impedance control as the problem to define a controller; therefore, the interaction forces govern the error between desired and actual positions of the end-effector. This is a very good definition of the problem, but it is mainly addressed to industrial robots. When robots are intended to interact with humans (i.e., service robots), the relationship between force and position should not only be accounted at the endeffector but the entire robot; otherwise, it might harm humans. Nevertheless, the principle of impedance control is the same for both industrial and service robotics. And it is a general approach to interaction control in which the robot behaves like a massspring-dapshot system with adjustable parameters. Therefore, the objective of impedance control is to make the interaction force to be dependent on the robot's position, velocity, and/or acceleration errors:

$$
F=M_{d}\left(\ddot{x}-\ddot{x}_{d}\right)+B_{d}\left(\dot{x}-\dot{x}_{d}\right)+K_{d}\left(x-x_{d}\right) .
$$

Note that (2) in the Laplace domain can be rewritten as

$$
F=Z_{d}(s)\left(X-X_{d}\right), \quad Z_{d}(s)=M_{d} s^{2}+B_{d} s+K_{d}
$$

where $Z_{d}(s)$ is the desired robot impedance; $M_{d}, B_{d}$, and $K_{d}$ are the desired apparent mass, damping, and stiffness matrices, respectively, which define the desired impedance; $F$ is the exerted force; $X$ is the actual robot position; and $X_{d}$ is the desired robot position. 
The impedance parameters can be selected in accordance to one or more tasks. The impedance control objective can be achieved using feedback control. However, in [22] it is established that impedance control can also be achieved without feedback control using kinematically redundant configurations. BigDog also uses directional compliance control at the distal limbs without feedback [13]. One advantage of using impedance modulation without feedback control is the avoidance of destabilizing effects due to transmission delays.

\section{IMPEDANCE CONTROL SCHEMES}

Impedance control is not a concrete control scheme, but a control strategy to solve the issues that arise when the robotenvironment interaction cannot be neglected (i.e., constrained motion). Although it can be achieved by many different control strategies, in robotic implementation, it is usually reduced to two basic procedures [23]. The first is a position-based approach, in which an inner position loop is implemented; the second is a force-based approach, in which an inner force loop is implemented.

\section{A. Position-Based Impedance Control}

In industrial robotics, the position-based approach has been traditionally implemented since industrial robots have high gear ratios, which implies nonlinear friction in the transmission system which complicates the force calculation for the joints. In addition, most of them already have implemented a position controller which is designed for path tracking. Moreover, adding a force sensor to the end-effector is very easy, and it does not modify the structure of the robot, while adding a force sensor on each joint implies modifying the robot's structure.

Fig. 1 shows a position-based impedance control scheme that is presented in [23], and it is referred as position-based impedance model error control by the authors. In this figure, $\mathbf{G}_{r}$, $\mathbf{G}_{e}$, and $\mathbf{C}_{p}$ are the robot, environment, and position controller transfer function, respectively. $\mathbf{G}_{f}$ is a force-feedback compensator which determines the relationship between the contact force and the position. In this case, the inner position loop is used to nullify the tracking error of $\Delta \mathrm{x}_{f}$ which is computed from the difference between the actual robot position and the reference position. Now, the reference position is computed from the desired position and the actual force multiplied by the force compensator $\mathbf{G}_{f}$, therefore reducing the difference between the plant behavior and the desired impedance.

The scheme presented previously and other position-based schemes, such as a generalization of the scheme proposed by Maples and Becker in [24], are explained in detail in [23].

\section{B. Force-Based Impedance Control}

Force-based impedance control, to be effectively implemented, needs either a force sensor at each joint or a predictable relationship between task space forces and the joint torques. These conditions are relatively easily met by low-geared [25] or direct-drive robots.

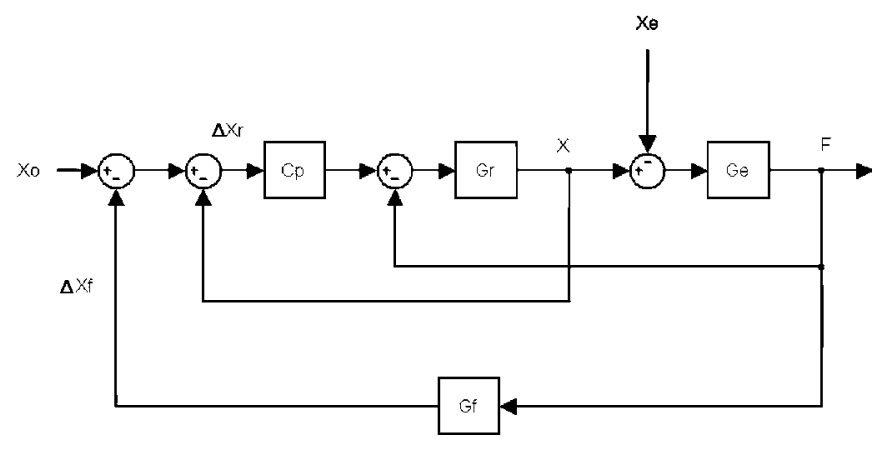

Fig. 1. Position-based impedance control.

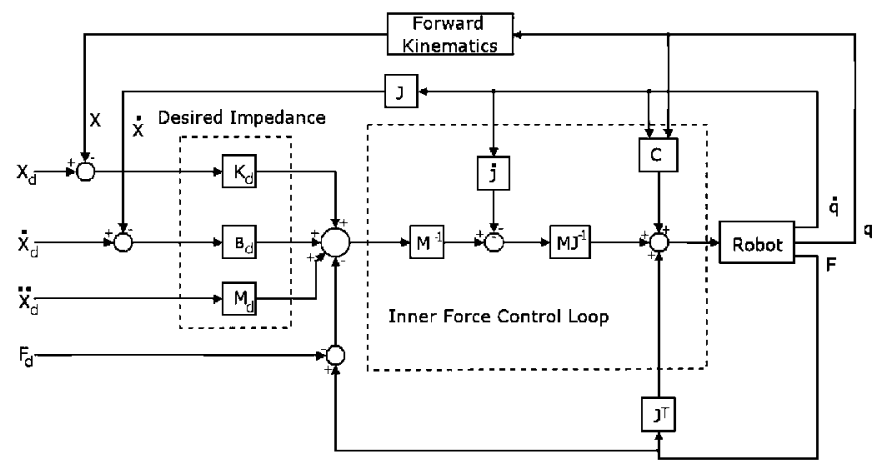

Fig. 2. Force-based impedance control.

Fig. 2 shows a force-based impedance control scheme. This scheme was originally proposed by Bonitz and Hsia [26] to cooperate manipulators, and it represents the following control law:

$$
\begin{aligned}
\tau= & \mathbf{M}\left[\mathbf{J}^{-1}\left(\mathbf{M}^{-} \mathbf{1}\left[\mathbf{M}_{\mathrm{d}} \ddot{\mathbf{x}}_{\mathrm{d}}+\mathbf{B}_{\mathrm{d}} \delta \dot{\mathbf{x}}+\mathbf{K}_{\mathrm{d}} \delta \mathbf{x}-\delta \mathbf{F}_{\mathbf{d}}\right]-\dot{\mathbf{J}} \dot{\mathbf{q}}\right)\right] \\
& +\mathbf{C}+\mathbf{J}^{\mathbf{T}} \mathbf{F}
\end{aligned}
$$

where $\mathbf{M}$ is the robot's inertia matrix; $\mathbf{C}$ is a matrix grouping Coriolis, centrifugal and gravitational terms; $\mathbf{J}$ is the robot's Jacobian matrix; $\mathbf{x}_{\mathrm{d}}$ is the desired trajectory; $\delta \mathbf{x}_{\mathbf{d}}=\mathbf{x}_{\mathbf{d}}-\mathbf{x}$ is the position tracking error; $\delta \mathbf{F}_{\mathrm{d}}=\mathbf{F}_{\mathrm{d}}-\mathbf{F}$ is the force tracking error; and $\mathbf{M}_{\mathrm{d}}, \mathbf{B}_{\mathrm{d}}$, and $\mathbf{K}_{\mathrm{d}}$ are the desired inertia, damping, and stiffness matrices, respectively, which define the robot's desired mechanical impedance.

The stability of this control law was studied in [26] using Lyapunov theory, stating that if $\mathbf{M}_{\mathrm{d}}, \mathbf{B}_{\mathrm{d}}$, and $\mathbf{K}_{\mathrm{d}}$ are symmetric positive definite and the Jacobian is nonsingular, the system is stable. Moreover, it is important to note that this control law is very similar to the one proposed by Hogan in [22].

Whether to choose force-based or position-based impedance controllers depends on what is needed. Lawrence [21] studied both implementations and demonstrated that position-based implementations are better suited to provide large impedances, while force-based implementations have difficulties in providing large ones. Moreover, he analyzed the effects of transmission delay in both options and established some boundaries for the systems' stability. This issue is addressed in the following sections. 


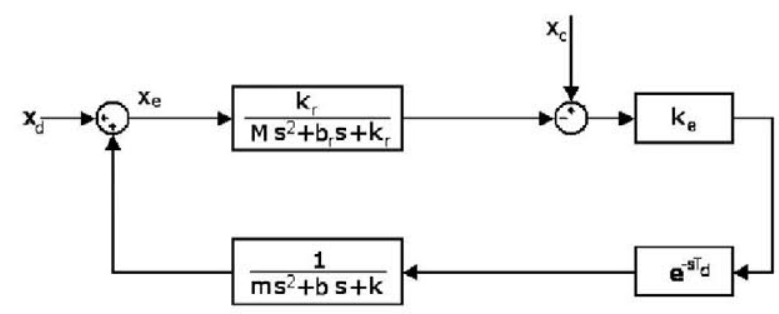

Fig. 3. Position-based impedance system model.

\section{Stability Boundaries for Impedance Control}

As stated before, impedance control has some stability problems to provide a certain range of impedances depending on whether it is a force-based or a position-based scheme. In order to analyze these problems, Lawrence [21] proposed two simple schemes.

The first scheme is a position-based impedance control along a single degree of freedom (DoF) (see Fig. 3), where $k_{r}, b_{r}$, and $M$ are the robot stiffness, damping, and mass, respectively; $k_{e}$ is the environment stiffness; and $m, b$, and $k$ are the desired mass, damping, and stiffness, respectively. The analysis of the system proposed by Lawrence was done with various assumptions, the most relevant being that the robot joints are decoupled and that the dynamic behavior of the robot is invariant [21]. These assumptions yield the following transfer function:

$$
\frac{x}{x_{e}}=-\frac{k_{r} k_{e} e^{-s T_{d}}}{\left(M s^{2}+b_{r} s+k_{r}\right)\left(j s^{2}+b^{s}+k\right)} .
$$

Analyzing the stability of the previous transfer function via phase margin gives the following relations for a stable control system:

$$
\begin{aligned}
k_{r} k_{e}= & \sqrt{\left[\left(k_{r}-\omega^{2} M\right)^{2}+b_{r}^{2} \omega^{2}\right]\left[\left(k-\omega^{2} m\right)^{2}+b^{2} \omega^{2}\right]} \\
-\pi= & -\tan ^{-1}\left(\frac{b_{r} \omega}{k_{r}-\omega^{2} M}\right)-\tan ^{-1}\left(\frac{b \omega}{k-\omega^{2} m}\right) \\
& -\omega T_{d} .
\end{aligned}
$$

These are a set of nonlinear equations in the frequency $\omega$ which can be solved by means of numerical methods, such as the Newton-Rhapson algorithm. Fig. 4 shows the solution considering $m=0, k_{r}=1000, b_{r}=100, M=20$, and $k_{e}=100$ and for various time delays $T_{d}$. These curves have been obtained via simulation, using different parameters.

The area below each curve corresponds to values for $b$ and $k$ which make the system unstable for the given delay $T_{d}$. As can be seen from the graph and in concordance with [21], even an ideal zero-delay $\left(T_{d}=0\right)$ position-based impedance controller cannot provide a full range of impedances, being impossible to obtain low stiffness and low damping at the same time for a stable performance.

The force-based impedance control system which is analyzed by Lawrence is presented in Fig. 5, and its transfer function is as follows:

$$
\frac{x}{x_{e}}=-\frac{\left(k+b s+m s^{2}\right) e^{-s T_{d}}}{m s^{2}} .
$$

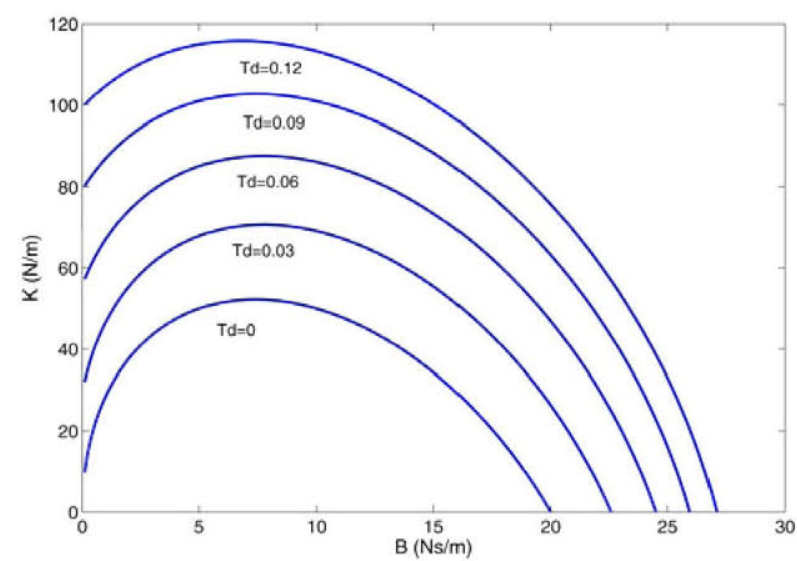

Fig. 4. Stability boundaries for the position-based impedance control for different time delays. System is unstable for $K$ and $B$ values below each curve.

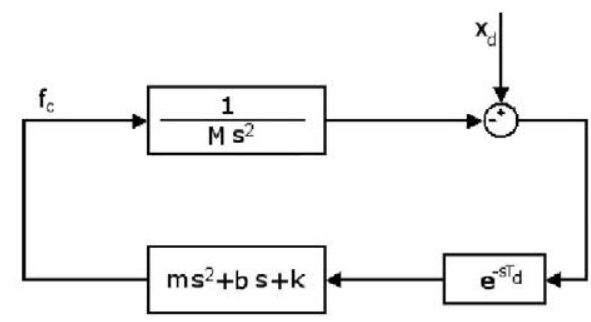

Fig. 5. Force-based impedance system model.

As for the position-based approach, this system is considered under various assumptions [21]. Note that the transfer function in this case is monotonically decreasing, which yields the following stability margin relations:

$$
\begin{aligned}
M \omega^{2} & =\sqrt{\left(k-m \omega^{2}\right)^{2}+b^{2} \omega^{2}} \\
0 & =\tan ^{-1}\left(\frac{b \omega}{k-m \omega^{2}}\right)-\omega T_{d} .
\end{aligned}
$$

These equations can also be numerically solved for any parameters. Fig. 6 shows the solutions for different time delays. In this graph, the area below each curve represents the stable values, contrary to the curves in Fig. 4. It is important to note that in this case, the equation set cannot be solved for an exact zero-delay force as a consequence of the tangential equation. However, it can be observed in the graph that as the delay decreases, the curve approximates the vertical; hence, an ideal zero-delay force-based impedance control is capable of providing the full range of impedances. However, as the delay increases, the force-based impedance control capability to provide large stiffness decreases.

The analysis performed by Lawrence, although it has many simplifications, was experimentally validated on an industrial robot for the position-based scheme. This analysis is very interesting since it provides a basic understanding on stability issues for impedance control and a guidance on whether to choose force-based or position-based schemes depending on the final application. 


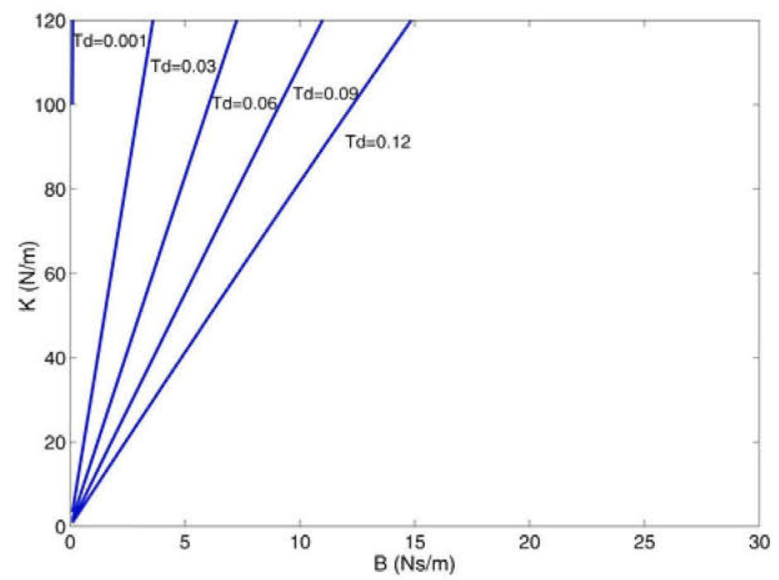

Fig. 6. Stability boundaries for the force-based impedance control different time delays. System is unstable for $K$ and $B$ values above each curve.

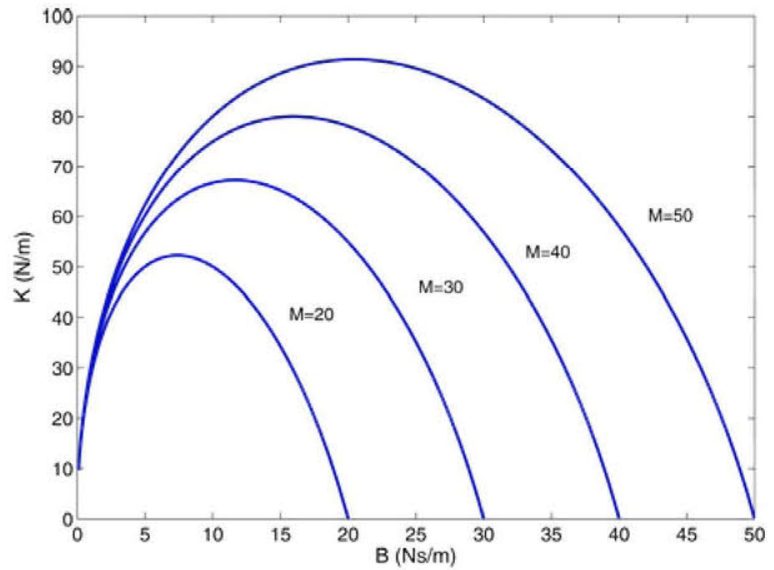

Fig. 7. Stability boundaries for the position-based impedance control with zero delay and different mass. Unstable region is below each curve.

The aforementioned analysis did not take into consideration the effect of robot mass in the stability boundaries. Such analysis is here presented, thus completing the stability boundaries study initiated by Lawrence. Analyzing the stability boundaries for both approaches with different robot masses provides an additional criteria to perform the selection between them. Fig. 7 shows the ideal zero-delay curve for $M=\{20,30,40,50\}$ for the position-based impedance control, and Fig. 8 shows the results when solving the equation with the same parameters for the force-based scheme.

As can be inferred from the curves, for the position-based approach, the unstable area widens as the mass increases; conversely, the opposite occurs for the force-based approach: the stable region widens as the mass increases. In Fig. 8, the scale of the axes has been extended to show the full range of the forcebased impedance control approach, and as it can be seen the stable region is much wider for small impedances. One implication of this analysis is that position-based impedance control performance decreases as the robot mass increases and the opposite is true for force-based approaches. This is a rule to be taken into consideration when choosing the appropriate control scheme for a given robot.

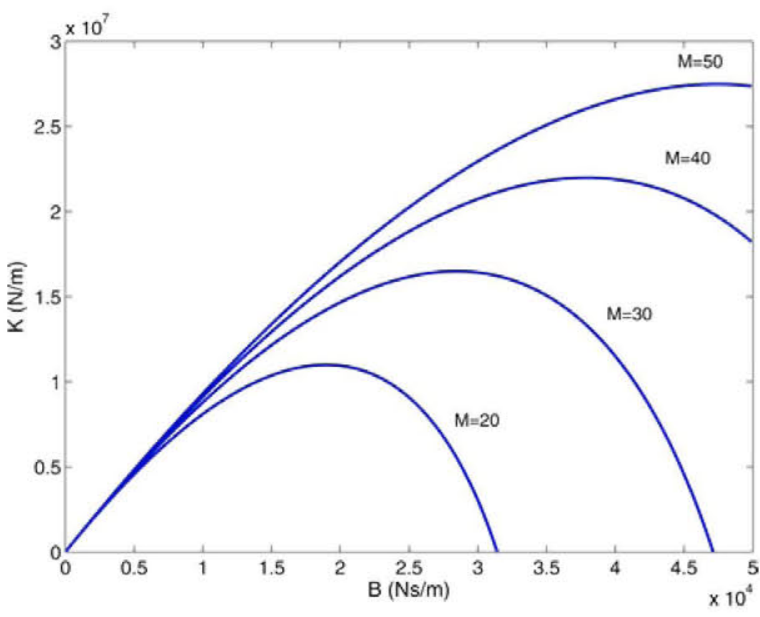

Fig. 8. Stability boundaries for the position-based impedance control with $T_{d}=0.001$ and different mass. Unstable region is above each curve.

\section{ENVIRONMENT/CONTACT MODELS}

A robot that is required to interact with its environment, and this interaction involves both contact and free space motion, can be modeled as a robot with an unequally constrained motion [27]. This means that when the distance between the robot and the contact surface is greater than zero, the reaction forces are zero and when the distance is equal to zero, the reaction force is not equal to zero.

The modeling of the aforementioned force is an important issue in robot-interaction tasks because, as stated in the previous section, the controlled robot's mechanical behavior should be chosen according to the environment with which it will be in contact.

First, let us establish the difference between contact and impact. Impact is a complex phenomenon that occurs when two or more bodies undergo a collision [28], and it is characterized by very short duration, rapid energy dissipation, large accelerations (positive and negative), and very high force levels. On the other hand, contact is a term which is used to describe situations where two or more bodies come in touch with each other, and it implies a continuous process that takes place in a finite time [28].

In the related literature, there are basically two types of approaches to describe impact. The first type is referred as impulse-momentum methods, and the second as force-based methods [29]. The first class of methods cannot be easily applied to robot-interaction tasks. This is because they are based on the perfect rigid body assumption, which in some cases, especially in impacts with very high velocities involved, is not true since it does not account for deformation. Yet another problem with impulse-momentum methods is that friction can only be taken into consideration using Coulmb's friction. The reason is that when analyzing impact with impulse-momentum methods, one can only obtain the velocity before and after the impact. More complete friction models describe friction in terms of a continuous velocity or contact force; however, they cannot be computed with impulse-momentum methods. For those reasons, we are interested in the force-based methods which generally describe 
the contact force (which includes impact force as well) as

$$
F_{c}=F_{v}(\dot{x})+F_{d}(x)
$$

where $F_{c}$ is a function of the local deformation, which is modeled as a displacement $x . F_{v}$ and $F_{d}$ are the reaction forces that are proportional to the indentation and the velocity of indentation, respectively. Within this class of methods, there are three main models in the literature.

1) Spring-dashpot model [30]

$$
F_{c}=b \dot{x}+k x .
$$

2) Hertz's model [31]

$$
F_{c}=F_{c, \max }\left(\frac{x-x_{p}}{x_{\max }-x_{p}}\right)
$$

where $F_{c, \max }$ and $x_{\max }$ are the maximum normal force and indentation, respectively, $x$ is the local indentation, and $x_{p}$ is the permanent indentation.

3) Nonlinear damping model [32], [33]

$$
F_{c}=b x^{p} \dot{x}^{q}+k x^{n} .
$$

In all the aforementioned equations, the velocity and force vectors are positive in the direction of the penetration of the robot into the environment.

In this paper, we are interested in the spring-dashpot model because it models impact forces for legged locomotion sufficiently well. However, for the interested reader the works of Giraldi and Dharf [28], Stewart [34], and Goldsmith [30] provide a good review of contact models, both impulse-momentum and force based.

In the spring-dashpot model, the contact force is modeled as a linear spring and a linear damper connected in parallel, in order to ensure elastic behavior and energy dissipation [30]. Therefore, the contact force is defined following (10).

According to Marhefka and Orin [35], this model has some weaknesses. First, the impact force is discontinuous due to the damping term, because at the instant of impact the velocity is different from zero. Second, as the objects separate, the relative velocity between them tends to be negative, and therefore, there is a negative force that holds the objects together. Finally, the coefficient of restitution (a quantity that expresses the energy loss caused by the impact), in this model, is independent from the impact velocity which was contradicted by experimental foundation [30].

Nevertheless, this model provides a reasonable method to capture energy dissipation associated with the normal forces without explicitly considering plastic deformation issues [28]. Moreover, many authors have successfully implemented this model [36]-[38] for manipulation tasks. For legged locomotion, Waldron et al. recently used them to analyze galloping and bounding [39].

\section{Model of A Robotic Leg in Contact WITH ITS ENVIRONMENT}

The aim of the previous section was to give a very brief overview on the contact models and explain why the contact

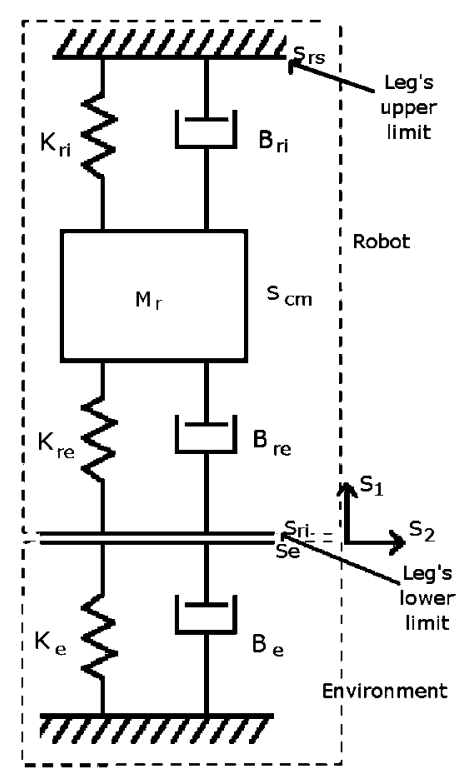

Fig. 9. Leg/environment contact model.

model used in this work was chosen. In this section, a model of a robot in contact with the environment using the springdashpot model is given. The environment can be any dynamic system working with the robot, i.e., another robot, a support, the ground, a rotating table, etc., as long as it can be described using this model.

In general, the dynamic model of a robot in joint coordinates is given by

$$
\mathbf{M}(\mathbf{q}) \ddot{\mathbf{q}}+\mathbf{C}(\mathbf{q}, \dot{\mathbf{q}})=\tau
$$

where $\mathbf{M}(\mathbf{q})$ is the inertia matrix, and $\mathbf{C}(\mathbf{q}, \dot{\mathbf{q}})$ is a matrix grouping the Coriolis, centrifugal and gravitational components.

Now, consider a robotic leg with $n$ DoF, which moves in the sagittal plane (the vertical plane which passes from front to rear dividing the body into right and left sections) and that is in contact with the environment. Such a leg can be described by the following expression:

$$
\mathbf{M} \ddot{\mathbf{q}}+\mathbf{C}=\tau+\mathbf{J}^{\mathrm{cT}} \mathbf{F}_{\mathrm{c}}+\mathbf{J}^{\mathrm{f}} \mathbf{F}_{\mathbf{f}}
$$

where $\mathbf{J}^{\mathrm{c}}$ is the Jacobian matrix in the constraint coordinate, $\mathbf{J}^{\mathbf{f}}$ is the Jacobian matrix in the free-motion coordinate, $\mathbf{F}_{\mathrm{c}}$ is the contact force, and $\mathbf{F}_{\mathrm{f}}$ is the friction force. Moreover, the foot is considered to be deformable, and the environment is modeled by the spring-dashpot contact model. The model of the foot is taken from [36], and it resembles the model used by Alexander in [40] to describe the paws in some mammals, such as dogs. The complete model is shown in Fig. 9.

Based on the assumption that the friction force is present in the directions where no deformation is allowed (i.e., the leg's walking direction), all the equations for the contact motion will be derived without the friction force and afterward the friction will be added. 


\section{A. Leg Model Without Friction}

In the model, the leg coordinates are referenced to a coordinate system that is fixed to the contact surface (see Fig. 9). If the surface and the robot were considered perfectly rigid, the contact is described by a geometrical constraint and it has the following form:

$$
\mathrm{s}^{\mathrm{c}}=0 .
$$

However, since the surfaces are assumed to be deformable, $\mathrm{s}^{\mathrm{c}}$ should describe the deformation along the coordinates where deformation is allowed. Nevertheless, when there is no contact force, (15) holds, but when the contact force exists, $\mathbf{s}^{\mathrm{c}}$ is described by

$$
\mathbf{s}^{\mathbf{c}}=\delta_{\mathrm{e}}+\delta_{\mathrm{ri}}+\delta_{\text {re }}
$$

where $\delta_{\mathrm{ri}}$ is the robot's internal deformation, $\delta_{\mathrm{re}}$ is the robot's external deformation, and $\delta_{\mathrm{e}}$ is the environment's deformation. The external deformation is the local deformation at the contact point, while the internal deformation is used to model the energy loss due to the transmission of stress waves in the body.

Now, the environment reaction force is given by the springdashpot model (10) and can be rewritten as

$$
\mathbf{F}_{\mathrm{e}}=\mathbf{k} \delta_{\mathrm{e}}+\mathbf{b} \dot{\delta}_{\mathrm{e}}
$$

considering that the environment has infinite mass after the spring-damper system. The relationship between the robot deformation and the joint motion can be described as

$$
\ddot{\mathbf{s}^{\mathrm{c}}}=\ddot{\delta}_{\text {ri }}+\ddot{\delta}_{\text {re }}=\mathbf{J}^{\mathrm{c}} \ddot{\mathbf{q}}+\dot{\mathbf{J}}^{\mathrm{c}} \dot{\mathbf{q}} .
$$

At this point, to completely describe the system (without including friction) the equations relating the environment's and robot's contact force are missing. These equations are the equation of motion for the robot's external deformation accelerations and the force equilibrium equation for the environment:

$$
\begin{aligned}
& \mathbf{F}_{\mathrm{e}}-\mathbf{F}_{\mathrm{c}}=\mathbf{0} \\
& \mathbf{M}_{\mathrm{r}} \ddot{\delta}_{\mathrm{re}}=-\left(\mathbf{F}_{\mathrm{c}}-\mathbf{F}_{\mathrm{c}_{\mathrm{re}}}\right) \\
& \mathbf{F}_{\mathrm{c}_{\mathrm{re}}}=-\mathbf{K}_{\mathrm{re}} \delta_{\mathrm{re}}-\mathbf{B}_{\mathrm{re}} \dot{\delta}_{\mathrm{re}} .
\end{aligned}
$$

And finally, the internal robot's deformation can be described by the following equation:

$$
\mathbf{F}_{\mathrm{c}_{\mathrm{ri}}}=-\mathbf{K}_{\mathrm{ri}} \delta_{\mathrm{ri}}-\mathbf{B}_{\mathrm{ri}} \dot{\delta}_{\mathrm{ri}} .
$$

Equations (14) through (22) define the system very well, and these equations can be solved for the robot's accelerations $\ddot{q}$ and forces $\mathbf{F}_{\mathrm{c}}$ with the exception of the friction force.

\section{B. Adding Friction}

Friction is a very complex phenomenon that occurs when two or more bodies are in contact and in relative motion. It depends on many parameters such as velocity, the material properties, temperature, etc. There has been extensive research effort in modeling friction; however, the models that are proposed concentrate on describing the main properties of the phenomenon.

As with contact, there are two main types of approaches to friction modeling: static and dynamic. Static models, due to their simplicity, are used in many applications [36], [41]; however, they do not explain several properties in systems with friction such as the Dahl effect, frictional lag, stick-slip motion, and presliding displacement [42] which can be relevant for certain applications and are considered in most dynamic models. See [43] and [44], which provide an insight in both static and dynamic friction models as well as friction compensation.

In this study, a modification of Coulomb's friction model is used, since it sufficiently describes the effects of friction; therefore, the friction force is as follows:

$$
F= \begin{cases}\mu_{d} F_{c} v, & \text { if } v \neq 0 \\ \mu_{s} F_{c}, & \text { if } v=0\end{cases}
$$

where $F$ is the friction force, $F_{c}$ is the contact force, and $v$ is a unit vector parallel to the relative velocity between the foot and the ground. $\mu_{c}$ is the Coulomb friction coefficient and $\mu_{s}$ is the static friction coefficient.

In order to include the friction force in the dynamic model of the robot in contact with the environment, what is left to define is the space where the friction force acts and relate it to the robot coordinates. Since friction exists in the direction of motion, the coordinates where friction occurs are unconstrained; the mapping between the work space and joint coordinates is as follows:

$$
\dot{\mathbf{s}}=\mathbf{J}^{f} \dot{\mathbf{q}}
$$

With this equation, the dynamics of the system can be modeled, since the unit vector $v$ depends on the direction of motion. It is important to note that if a constant friction coefficient $\mu$ is used, the forces are assumed to always be greater than the friction force. For non-constant coefficients when the relative velocity between the robot and the environment is zero, it is necessary to introduce the following condition [36]:

$$
\ddot{\mathbf{s}}^{f}=\mathbf{J}^{f} \ddot{\mathbf{q}}+\dot{\mathbf{J}}^{f} \dot{\mathbf{q}}
$$

This scenario is especially useful for walking robots, since it is essential that no relative motion between the foot and the environment during the support phase exists for the leg to propel the body during walking.

\section{IMPEDANCE CONTROL IN LEGGED LOCOMOTION}

The basic difference between controlling an industrial robot and a legged robot is the fact that, in legged robots, the robot's base is not fixed. Both the industrial and legged robot are considered to have its base at the body; however, in a legged robot, since it is a walking machine, there is no rigid connection between the base and a fixed coordinate frame [45]. Therefore, a common approach is to have an absolute coordinate frame on the ground and to use robot coordinates relative to that frame. Another important difference is that a walking robot is constantly changing from an open to a closed kinematic chain and vice versa, thus changing its $\mathrm{DoF}$. Moreover, for the robot to be able to walk it is necessary that no slip occurs when the feet are in contact with the ground, to propel the body. In order to meet that condition, it is necessary that the tangential forces do not overcome the static friction. That condition could be met 


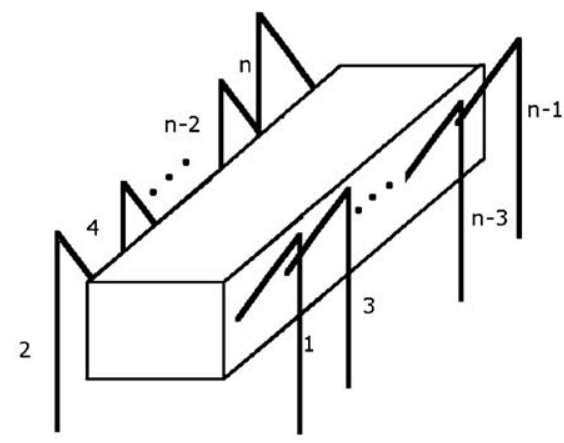

Fig. 10. A general legged robot with $n$ legs.

without force control; however, when the robot is intended to walk in complex terrain, the necessity to use force control so that contact forces are within friction cones may arise [16].

Direct force control is useful to improve the adaptation to terrain roughness [46]. Nevertheless, since walking robot legs have essentially two phases, one support phase (when the leg is in contact with the ground) and one transfer phase (when the leg is moving freely), force control cannot be used during the swing phase. Thus, it is necessary to either switch to a position controller during the transfer phase and then switch back to a force control approach when the leg is again in contact with the ground, or use some kind of impedance control strategy.

Impedance control has some advantages over direct force control in legged locomotion. Added to all what has already been presented trough this paper, it is obvious that when the foot hits the ground, an impact occurs. That impact can produce bouncing at the foot yielding to contact loss. This is a very important problem for multilegged robots, and specially for bipeds.

In this section, the characteristics of impedance control for legged locomotion are discussed. First, let us consider a generic walking robot with $n$ legs having $\frac{n}{2}$ legs on each side of its body (see Fig. 10) and assuming that at least $m$ legs are in support phase (with $m \leq n$ ), focusing on the two phases of the general gait pattern. Now, for each leg we have

$$
\begin{aligned}
& \mathbf{H}_{\mathrm{i}} \ddot{\mathbf{q}}_{\mathrm{i}}+\mathbf{G}_{\mathrm{i}} \ddot{\mathbf{x}}_{0}+\mathbf{J}_{\mathrm{i}}^{\mathrm{T}} \mathbf{F}_{\mathrm{i}}+\mathbf{C}_{\mathbf{i}}=\tau_{\mathrm{i}} \\
& \mathbf{Q} \ddot{\mathbf{q}}+\mathbf{R} \ddot{\mathbf{x}}_{0}+\mathbf{J F}+\mathbf{g}=\mathbf{0} .
\end{aligned}
$$

Here, $\mathbf{H}_{\mathbf{i}}$ and $\mathbf{G}_{\mathbf{i}}$ are the inertia matrices for each leg, where $\mathbf{H}_{\mathbf{i}}$ is the inertia of the leg itself, and $\mathbf{G}_{\mathbf{i}}$ is the body inertia seen from the $i$ th leg; $\mathbf{R}$ and $\mathbf{Q}$, on the other hand, are the inertia matrices for the body, where $\mathbf{R}$ is the body's inertia and $\mathbf{Q}$ is a row vector with the inertias of each leg seen from the body; $\mathrm{x}_{0}$ is the position/orientation of the body; $\mathrm{q}_{\mathrm{i}}$ is the position/orientation of the $i$ th leg; $\mathbf{J}_{\mathbf{i}}$ is the Jacobian of the $i$ th leg; and $\mathbf{J}$ is a row vector with the Jacobian of each leg in each column. For all the matrices, $i=1, \ldots, n$.

As can be deduced from the equations, controlling a walking robot is a very complex task. For that reason, the typical control strategy to follow is to divide the control system into two levels [45].

1) An upper level: it is responsible for gait pattern generation, computing of the foot velocities necessary to have a certain linear speed at the center of mass, computing the desired contact forces at each leg, etc.

2) A lower level: it is responsible to track the commands that are generated from the upper level.

The upper level is also responsible to assign the contact forces; these forces have to be of the appropriate value so that the robot can support the weight of its body and to ensure the stability of the robot. To do that, it is necessary to consider how many legs are in support phase and the weight distribution among the legs, because each leg does not need to support the same weight and that depends on their distribution (e.g., the weight distribution is not the same if the legs are distributed in a rectangular pattern than in a circular pattern). The interested reader can find very interesting works on force distribution in the literature of the field; representative works include [47] and [46] where hexapod robots are analyzed, and the work of Yoneda et al. [17] where a quadruped is studied. Besides, the decision on force distribution in dynamic walkers should be linked to the control of dynamic stability. The stability in legged locomotion is acquired via several stability criteria, although research on bipeds concentrates on the use of Zero Moment Point (ZMP) [48] and now the Centroidal Moment Pivot (CMP) [49]. Find a comprehensive survey in [50]. The simultaneous control of impedance and dynamic stability has been addressed in [20].

Here, our primary concern is to establish an impedance control law which is the work of the lower level. It is necessary to have knowledge about the leg's phase, because the programmed impedance should not be the same in the transfer phase than in the support phase.

\section{A. Leg Support Phase}

As mentioned before, we assume that $m$ legs are in support phase simultaneously. The actual value of $m$ depends on the gait pattern. It is important to note that the gait pattern generation is not a concern of this study. However, in general

$$
\frac{n}{2} \leq m \leq n .
$$

Regarding the foot force, the vertical component should support the robot's weight and payload, and the horizontal components need to be less than the static friction to allow propulsion. Now, assuming that the appropriate force reference value from the upper level controller is obtained, each leg behavior can be controlled according to one of the two control schemes that are presented in section III. The support phase starts when the leg initiates contact with the ground; ergo, an impact occurs. To minimize the impact force, it is necessary that the foot impedance has high damping and low stiffness at the moment of contact in order to counteract the impact force. Afterward, it is desired to increase the stiffness gradually when the leg is supporting the corresponding fraction of the robot's weight [51]. This increase in leg stiffness is required because supporting a robot's weight generally implies a high force and having low stiffness will imply a high position deviation, which is undesirable in order to maintain the robot configuration constant. For that reason, the force-based approach is more suitable [see (4)]. 
The desired leg impedance is described by the following equation:

$$
\begin{aligned}
\mathbf{Z} & =\mathbf{M}_{\mathrm{di}}\left(\ddot{\tilde{\mathbf{x}}_{\mathbf{i}}}\right)+\mathbf{B}_{\mathrm{di}}\left(\dot{\hat{\mathbf{x}}}_{\mathbf{i}}\right)+\mathbf{K}_{\mathrm{di}}\left(\hat{\mathbf{x}}_{\mathbf{i}}\right) \\
\hat{\mathbf{x}}_{\mathbf{i}} & =\mathbf{x}_{\mathbf{i}}-\mathbf{x}_{\mathrm{di}}
\end{aligned}
$$

where $\mathbf{x}_{\mathbf{i}}$ is the foot position; $\mathbf{x}_{\mathrm{di}}$ is the desired foot position, which is also provided by the upper control level; $\hat{\mathbf{x}}_{\mathbf{i}}$ is the position error; and $\mathbf{M}_{\mathrm{di}}, \mathbf{B}_{\mathrm{di}}$, and $\mathbf{K}_{\mathrm{di}}$ are the inertia, damping, and stiffness matrices that describe the $i$ th leg impedance, respectively. Now, combining (26) with (29) yields

$$
\begin{aligned}
\tau= & \mathbf{H}_{\mathbf{i}}\left[\mathbf { J } _ { \mathrm { i } } ^ { - 1 } \left(\mathbf { H } _ { \mathrm { i } } ^ { - } \mathbf { 1 } \left[\mathbf{M}_{\mathrm{di}} \ddot{\mathbf{x}}_{\mathrm{di}}+\mathbf{B}_{\mathrm{di}} \dot{\hat{\mathbf{x}}}_{\mathbf{i}}+\mathbf{K}_{\mathrm{di}} \hat{\mathbf{x}}_{\mathbf{i}}\right.\right.\right. \\
& \left.\left.\left.-\hat{\mathbf{F}}_{\mathrm{i}}\right]-\dot{\mathbf{J}}_{\mathrm{i}} \dot{\mathbf{q}}_{\mathbf{i}}\right)\right]+\mathbf{C}^{*}+\mathbf{J}_{\mathrm{i}}^{\mathrm{T}} \mathbf{F}_{\mathrm{i}} \\
\mathbf{C}^{*}= & \mathbf{C}+\mathbf{G}_{\mathrm{i}} \ddot{\mathbf{x}}_{0} \\
\hat{\mathbf{F}}_{\mathrm{i}}= & \mathbf{F}_{\mathbf{i}}-\mathbf{F}_{\mathrm{di}}
\end{aligned}
$$

where $\mathbf{F}_{\mathrm{i}}$ is the contact force, which is described by the contact model; $\mathbf{F}_{\mathrm{di}}$ is the desired contact force; $\hat{\mathbf{F}}_{\mathrm{i}}$ represents the force tracking error; $\mathbf{J}_{\mathbf{i}}$ is the Jacobian matrix for the $i$ th leg; $G_{i}$ is the reflected body inertia seen from the $i$ th leg; and $\ddot{\mathbf{x}}_{0}$ is the center of mass acceleration.

It is important to note that as mentioned before the desired contact force should be chosen to be less than the static friction, that is, if we consider

$$
\begin{aligned}
& \mathbf{x}_{\mathbf{i}}=\left\{x_{i}, y_{i}, z_{i}\right\} \\
& \mathbf{F}_{\mathbf{i}}=\left\{F_{i}^{x_{i}}, F_{i}^{y_{i}}, F_{i}^{z_{i}}\right\}
\end{aligned}
$$

where $z_{i}$ is the vertical component, the contact force in the directions $x_{i}$ and $y_{i}$ should satisfy the following set of equations:

$$
\begin{aligned}
F_{i}^{x_{i}} & \leq k \mu_{s}\left|F_{i}^{z_{i}}\right| \\
F_{i}^{y_{i}} & \leq k \mu_{s}\left|F_{i}^{z_{i}}\right|
\end{aligned}
$$

where $\mu_{s}$ is the static friction coefficient of the static friction; and $k$ is an nondimensional constant which is chosen as a safety factor. For example, in [47] this factor is chosen to be $\sqrt{(2) / 2}$ which is a conservative solution; however, the limit condition is $k=1$.

\section{B. Leg Transfer Phase}

During the transfer phase, two facts should be considered: first, it is desirable that the foot position follows the prescribed trajectory as close as possible, and second, that the leg motion is now unconstrained. For that reason, the desired reaction force should be set to zero. Setting the desired reaction force to zero and assuming that since the leg is not in contact with the environment the contact force is equal to zero yields the following control law for each leg:

$$
\tau=\mathbf{H}_{\mathrm{i}}\left[\mathbf{J}_{\mathbf{i}}^{-1}\left(\mathbf{H}_{\mathrm{i}}^{-1}\left[\mathbf{M}_{\mathrm{di}} \ddot{\mathbf{x}}_{\mathrm{di}}+\mathbf{B}_{\mathrm{di}} \dot{\hat{\mathbf{x}}}_{\mathbf{i}}+\mathbf{K}_{\mathrm{di}} \hat{\mathbf{x}}_{\mathbf{i}}\right]-\dot{\mathbf{J}}_{\mathrm{i}} \dot{\mathbf{q}}_{\mathbf{i}}\right)\right]+\mathbf{C}^{*}
$$

where all the terms are the same as in (32). This control law, as can be deduced by the equation, is equivalent to a plus differential position controller with dynamic compensation. This means that the position can be effectively controlled with the

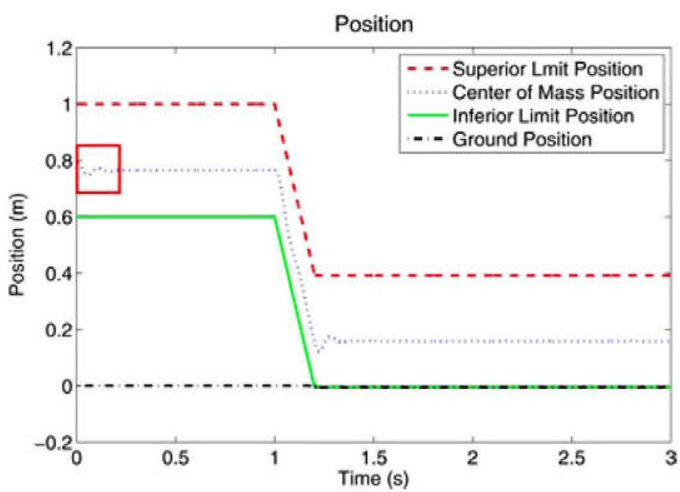

Fig. 11. Leg's upper limit (dashed line), inferior limit (continuous line), center of mass (dotted line), and environment (dash-dotted line) positions for an openloop interaction.

same control law. As for the desired impedance, the stiffness should be decreased, and the damping should be increased in order to prepare the leg for the next stance phase.

\section{Simulation of a Robotic Foot in CONTACt WITH ITS ENVIRONMENT}

A simulation to illustrate the previously described concepts is here presented. First, let us reconsider the foot presented in Fig. 9, where $s_{1}$ and $s_{2}$ are the unit vectors of the coordinate frame, and they are fixed on the contact surface of the environment; $s_{r i}$ and $s_{r e}$ are the coordinates of the leg's superior limit and the leg's inferior limit (foot), respectively, $s_{\mathrm{cm}}$ is the position of the center of mass of the leg; and $s_{e}$ is the position of the environment.

Selecting $M_{r}=5 \mathrm{~kg}, B_{r i}=50 \mathrm{Ns} / \mathrm{m}, K_{r i}=7000 \mathrm{~N} / \mathrm{m}$, $B_{r e}=100 \mathrm{Ns} / \mathrm{m}, K_{r e}=10000 \mathrm{~N} / \mathrm{m}, B_{e}=50 \mathrm{Ns} / \mathrm{m}$, and $K_{e}=$ $5000 \mathrm{~N} / \mathrm{m}$ yields the robot's leg to be more rigid than the environment which corresponds to the general case of a legged robot on a natural ground. The leg length is considered to be $0.4 \mathrm{~m}$, which is considered to be an average value of a leg length for a medium-sized legged robot. The equation of motion of the leg in equilibrium is presented here:

$$
0=K_{r i}\left(S_{r s}-S_{\mathrm{cm}}\right)+K_{r e}\left(S_{r i}-S_{\mathrm{cm}}\right) .
$$

The value of the spring constants and the leg's center of mass position are determined by this equation. Fixing the spring constants has a direct influence on the center of mass position, and the inverse also holds. Fixing the center of mass position, the relationship of the two spring constants is determined. Following this explanation, we assume that the center of mass is placed at $S_{\mathrm{cm}}=S_{r s}-0.2$ which means that the center of mass is in the midpoint between the lower and the upper limits of the leg. In order to show the behavior of the system in the case that the spring constants are not chosen according to (39), the spring constant for the internal deformation was chosen a bit smaller than the value that is obtained from the equation. This effect is an oscillation caused because the system naturally tends to the equilibrium, and it is shown in the rectangle in Fig. 11.

During the simulation process, the leg is moved along the coordinate $s_{1}$ until it makes contact with the environment. 


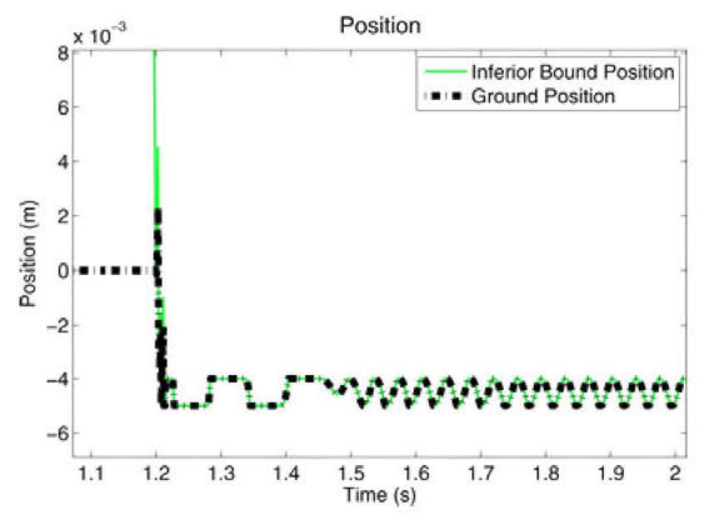

Fig. 12. Leg's inferior limit (continuous line) and environment (dash-dotted line) for an open-loop interaction.

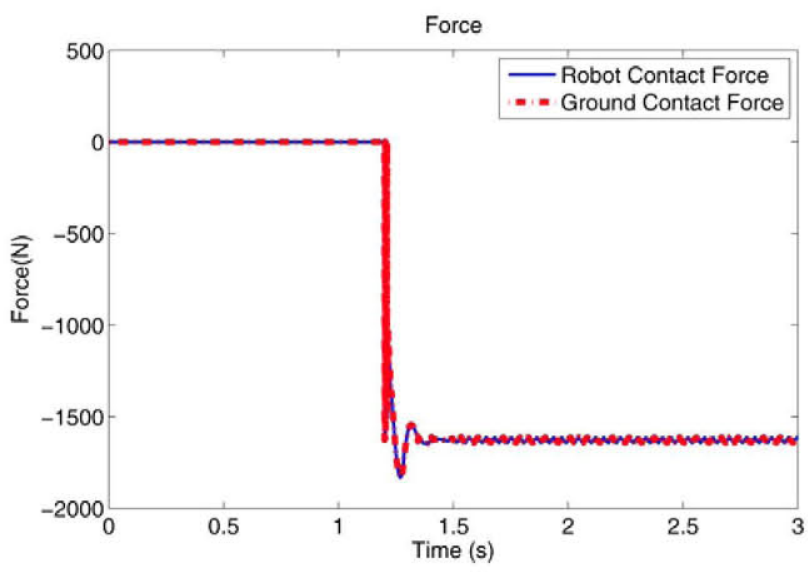

Fig. 13. Foot contact force (continuous line) and environment contact force (dash-dotted line) for an open-loop interaction.

Afterward, the foot keeps pushing against the environment until the end of the simulation. Fig. 11 shows the position of the leg's upper limit (dashed line), the postion of the leg's lower limit (continuous line), the position of the leg's center of mass (dotted line), and the environment's position (dash-dotted line) versus time in open loop. In this figure, it can be observed that at $t=1.2 \mathrm{~s}$ the foot makes contact with the ground which induces a deformation and initially the ground and the leg lose contact in two occasions; this effect is shown in Fig. 12, which is a zoomed version of Fig. 11 at $t=1.2 \mathrm{~s}$ for the inferior limit position curve. Additionally, Fig. 12 shows that even though the contact is maintained the position exhibits a chattering behavior, because both objects (the foot and the ground) keep pushing merely reacting to one another since there is no feedback to maintain a constant value of force in the leg.

Fig. 13, on the other hand, shows the leg's contact force (continuous line) and the ground contact force (dash-dotted line). In this figure, it is also observed that the foot makes contact with the ground at $t=1.2 \mathrm{~s}$, and the impact force generates the large peaks in the force curve. In this case, the initial peak was smaller than the second, but this is not necessarily always true; it depends on the elastic coefficients of both the robotic leg and the ground. Moreover, the chattering behavior that is observed in Fig. 12 is also present here. This chattering behavior will be corrected when feedback impedance control is introduced.

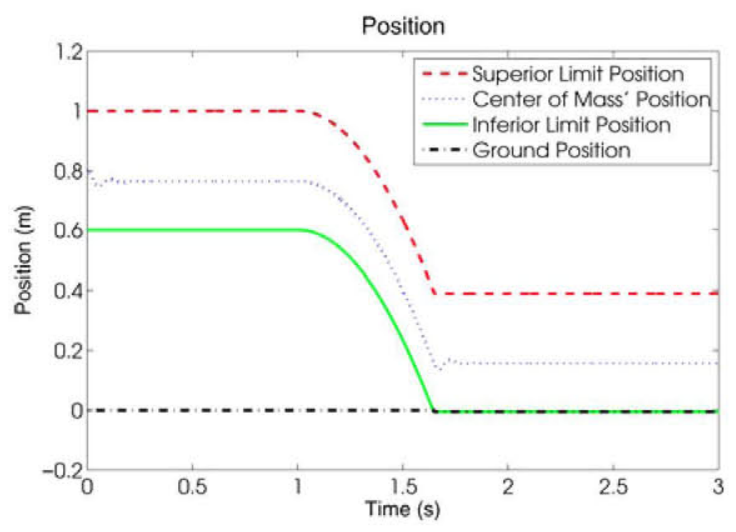

Fig. 14. Leg's upper limit (dashed line), inferior limit (continuous line), center of mass (dotted line), and environment (dash-dotted line) positions for a feedback impedance control of interaction.

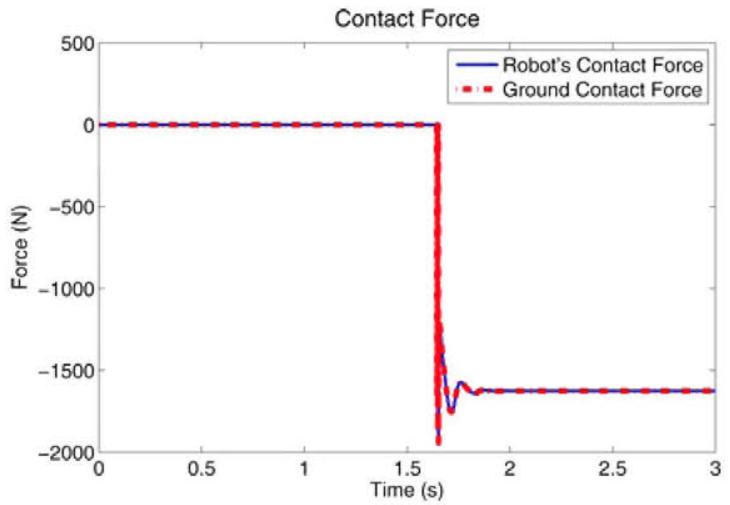

Fig. 15. Foot contact force (continuous line) and environment contact force (dash-dotted line) (closed loop).

In order to improve the interaction between the leg and the ground, a feedback impedance control scheme is applied. The control law that is applied to the system is the one described by (4) considering the task coordinates to be the foot position and the joint coordinates to be the leg's upper limit position, because it is considered to be a prismatic joint, and selecting the desired impedance constants to be $B_{d}=5 \mathrm{Ns} / \mathrm{m}, K_{d}=5 \mathrm{~N} / \mathrm{m}$, and $M_{d}=0 \mathrm{~kg}$. Fig. 14 shows the position of the leg's upper limit (dashed line), the postion of the leg's lower limit (continuous line), the position of the leg's center of mass (dotted line), and the environment's position (dash-dotted line) versus time in a closed loop. The desired position which is introduced in the control law is the same as the position input for the system in an open loop. However, because the desired force is set to zero, the trajectory of the leg is nonlinear in this case. In this figure, it can also be observed that the foot makes contact with the ground at $t=1.64 \mathrm{~s}$, and it also shows that the chattering behavior is corrected.

Fig. 15 shows the leg's contact force (continuous line) and the ground contact force (dash-dotted line). In this figure, the impact force is also observed with an initial peak that is higher in magnitude than the peak in Fig. 13; this could be corrected by changing the selected desired impedance. Nevertheless, the second peak is smaller than the second peak in Fig. 13, and the chattering behavior is also corrected, which makes the contact more stable. 


\section{CONCLUSION}

With the evolution of technology, the applications of robotic research have been broadened moving from only aiding humans in repetitive tasks to assisting them in more complex tasks. Most of these modern applications involve contact with an unstructured environment and with humans, therefore imposing the need to use an interaction control strategy, such as impedance control.

Impedance control has been extensively studied and successfully implemented in the past. However, the new applications of robotics such as IADs, agile, walkers and exoskeletons have different requirements in the interaction with the environment. In order to learn how to adapt impedance control to fit the new requirements, it is necessary to study the stability properties of its two main approaches of impedance control, along with the concepts of contact and friction. The aim of this paper is to provide an insight on these concepts and a guide on how to adapt them to new robotic applications by analyzing the case of legged locomotion.

Impedance control has two main approaches: force-based and position-based schemes. These approaches have different capabilities, and the selection between them must be performed according to the application. As has been shown here, positionbased impedance control cannot provide small impedances, i.e., low stiffness and low damping even with ideal zero delay; in addition, it is better suited for small robot mass since its stable region widens as the mass decreases. This paper has also shown that force-based impedance control is capable of providing the full range of impedances with zero delay; however, contrary to the position-based approach, as the mass increases, its stable region increases. This can be advantageous for medium- to large-sized legged robots. Therefore, when small impedance is required, it is better to use the force-based approach, and when the robot mass is small, the position-based approach works better than the force-based approach for large impedances under the same delay conditions.

For walking robots, impedance control can further increase the terrain adaptability of the robots. Nevertheless, when controlling a walking robot, and in general, any robot in contact with the environment, friction and contact forces should be taken into account. Moreover, the vertical component of the contact force should be controlled to ensure that the robot adequately supports its own weight, not to mention that the impact forces that occur at the instant of contact should be reduced, especially in the case of bipeds, because they can destabilize the robot.

Contact models should describe the contact force continuously, which is the case of dynamic models, such as the springdashpot model, which completely describes the impact force and accurately models energy losses due to this phenomenon.

Regarding friction, for the robot to be able to move, the leg must not slip. Because of this, in the case of walking robots, simple models are enough because the only requirement in order to ensure this condition is that the tangential contact forces need to be restrained within friction cones (limited by static friction).

Impedance control is beneficial during the gait cycle. At the beginning of the support phase, the damping should be high, and the stiffness should be low in order to reduce the bouncing produced by the impact. It is important to note that this impact can be seen as a disturbance for the control law, but as said before it is desirable to minimize the bouncing effect. Then, the stiffness should be increased in order for the robot to be able to maintain its position without significant deviation. During the transfer phase, the impedance should go back to have low stiffness and high damping in order to prepare it for the upcoming support phase. The best impedance control approach to implement this strategy is the force-based impedance control since it provides a wider range of impedances than the position-based approach.

A simulation of a leg using the robot environment model proposed in section $\mathrm{V}$ is presented. The simulation results illustrate the difference between the impedance-based controlled and uncontrolled systems under an impact event, showing that the contact when the control law is applied is smoother.

Future work includes the implemenatation of the models proposed in this paper on a three-DoF leg prototype.

\section{REFERENCES}

[1] A. B. Zoss, H. Kazerooni, and A. Chu, "Biomechanical design of the Berkeley lower extremity exoskeleton (BLEEX)," IEEE/ASME Trans. Mechatronics, vol. 11, no. 2, pp. 128-138, Apr. 2006.

[2] S. Lee and Y. Sankai, "Power assist control for walking aid with HAL-3 based on EMG and impedance adjustment around knee joint," in Proc. Int. Conf. Intell. Robots Syst., 2002, pp. 1499-1504.

[3] K. H. Low, X. Liu, and H. Yu, "Design and implementation of NTU wearable exoskeleton as an enhancement and assistive device," Appl. Bion. Biomech., vol. 3, no. 3, pp. 209-225, 2006.

[4] C. J. Walsh and K. Endo, "A quasi-passive leg exoskeleton for loadcarrying augmentation," Int. J. Human. Robot., vol. 4, pp. 487-506, 2007.

[5] J. Rosen, M. Brand, M. B. Fuchs, and M. Arcan, "A myosignal-based powered exoskeleton system," IEEE Trans. Syst., Man, Cybern. A, Syst., Humans, vol. 31, no. 3, pp. 210-222, May 2001.

[6] C. Fleischer and G. Hommel, "A human exoskeleton interface utilizing electromyography," IEEE Trans. Robot., vol. 24, no. 4, pp. 872-882, Aug. 2008.

[7] M. Raibert, K. Blankespoor, G. Nelson, and R. Playter, "BigDog, the rough-terrain quadruped robot," presented at the 17th World Congr. Int. Federation of Automatic Control, Seoul, Korea, 2008.

[8] J. Estremera and K. Waldron, "Thrust control, stabilization and energetics of a quadruped running robot," Int. J. Robot. Res., vol. 27, no. 10, pp. $1135-1151,2008$.

[9] C. Semini, "HyQ-Design and development of a hydraulically actuated quadruped robot," Ph.D. dissertation, Italian Instit. Technol. and Univ. Genoa, 2010.

[10] E. Garcia, J. Arevalo, G. Munoz, and P. Gonzalez-de-Santos, "Combining series-elastic actuation and magneto-rheological damping for the control of agile locomotion," Robot. Auton. Syst., vol. 50, no. 10, pp. 827-839, 2011.

[11] D. Surdilovic, R. Bernhardt, and L. Zhang, "New intelligent power-assist systems based on differential transmission," Robotica, vol. 21, pp. 295$302,2003$.

[12] A. Loredo-Flores, E. Gonzalez-Galvan, J. Cervantes-Sanchez, and A. Martinez-Soto, "Optimization of industrial, vision-based, intuitively generated robot point-allocating tasks using genetic algorithms," IEEE Trans. Syst., Man, Cybern. C, Appl. Rev., vol. 38, no. 4, pp. 600-608, Ju1. 2008.

[13] V. Lee and A. A. Biewener, "Bigdog-inspired studies in the locomotion of goats and dogs," J. Integrat. Comparat. Biol., vol. 51, no. 1, pp. 190-202, 2011.

[14] L. Sciavicco and B. Siciliano, Modelling and Control of Robot Manipulators, 2nd ed. Great Britain: Springer-Verlag, 2001.

[15] A. Schneider and U. Schmucker, "Force legged platform "katharina" for service operations," in Proc. 4th Int. Conf. Climb. Walking Robots, 2001, pp. $1029-1036$.

[16] D. M. Gorinevsky and A. Y. Shneider, "Force control in locomotion of legged vehicles over rigid and soft surfaces," Int. J. Robot. Res., vol. 2, no. 9, pp. 4-23, Apr. 1990. 
[17] K. Yoneda, H. Iiyama, and S. Hirose, "Sky-hook suspension control of a quadruped walking vehicle," in Proc. IEEE Int. Conf. Robot. Autom., May, 1994, vol. 2, pp. 999-1004.

[18] J. Buchli, M. Kalakrishnan, M. Mistry, P. Pastor, and S. Schaal, "Compliant quadruped locomotion over rough terrain," in Proc. IEEE/RSJ Int. Conf. Intell. Robots Syst., St. Louis, MO, 2009, pp. 814-820.

[19] Q. Huang, "Softly stable walk using phased compliance control with virtual force for multi-legged walking robot," in Proc. Int. Conf. Climb. Walk. Robots, 2010, pp. 1-18.

[20] E. Garcia and P. Gonzalez-de-Santos, "On the improvement of walking performance in natural environments by a compliant adaptive gait," IEEE Trans. Robot., vol. 22, no. 6, pp. 1240-1253, Dec. 2006.

[21] D. Lawrence, "Impedance control stability properties in common implementations," in Proc. IEEE Int. Conf. Robot. Autom., Apr. 1988, vol. 2, pp. 1185-1190.

[22] N. Hogan, "Impedance control: An approach to manipulation. Part Itheory. Part II-Implementation. Part III-Applications," J. Dyn. Syst., Meas., Control, vol. 107, pp. 1-7, Mar. 1985.

[23] M. Vukobratovic, D. Surdilovic, Y. Ekalo, and D. Katic, Dynamics and Robust Control of Robot-Environment Interactio. (New Frontiers in Robotics), vol. 4. Singapore: World Scientific, 2009.

[24] J. A. Maples and J. J. Becker, "Experiments in force control of robotic manipulators," in Proc. IEEE Int. Conf. Robot. Autom., 1986, vol. 2, pp. 695-703.

[25] D. Vischer and O. Khatib, "Design and development of high-performance torque-controlled joints," IEEE Trans. Robot. Autom., vol. 11, no. 4, pp. 537-544, Aug. 1995.

[26] R. G. Bonitz and T. C. Hsia, "Internal force-based impedance control for cooperating manipulators," IEEE Trans. Robot. Autom., vol. 12, no. 1, pp. 78-89, Feb. 1996.

[27] R. Featherstone, Rigid Body Dynamics Algorithms. New York: Springer-Verlag, 2008.

[28] G. Giraldi and I. Dharf, "Literature survey of contact dynamics modelling," Mech. Mach. Theory, vol. 37, pp. 1213-1239, 2002.

[29] S. Kim, "Contact dynamics and force control of flexible multi-body systems," Ph.D. dissertation, McGill Univ., Montreal, 1999.

[30] W. Goldsmith, Impact: The Theory and Physical Behavior of Colliding Solids. London, U.K.: E. Arnold, 1960.

[31] S. Abrate, Impact on Composite Structures. Cambridge, U.K.: Cambridge Univ. Press, 1998.

[32] K. H. Hunt and F. R. E. Crossley, "Coefficient of restitution interpreted as damping in vibroimpact," J. Appl. Mech., vol. 42, pp. 440-445, 1975.

[33] H. Hertz, D. E. Jones, and G. A. Schott, Miscellaneous Papers. London, U.K.: Macmillan, 1896.

[34] D. Stewart, "Rigid-body dynamics with friction and impact," SIAM Rev, vol. 42 , no. 1 , pp. 3-39, 2000.

[35] D. Marhefka and A. Orin, "A compliant contact model with nonlinear damping for simulation of robotic systems," IEEE Trans. Syst., Man, Cybern. A, Syst. Humans, vol. 6, no. 29, pp. 566-572, Nov. 1999.

[36] M. Vukobratovic and V. Potjonjak, "Dynamics of contact tasks in robotics: Part I. General model of robot interacting with environment," Mech. Mach. Theory, vol. 34, pp. 923-942, 1999.

[37] W. Howard and V. Kumar, "A minimum principle for the dynamic analysis of systems with frictional contacts," in Proc. IEEE Int. Conf. Robot. Autom. 1, 1993, pp. 437-442.

[38] K. Mirza, M. Hanes, and D. Orin, "Dynamic simulation of enveloping power grasps," in Proc. IEEE Int. Conf. Robot. Autom. 1, 1993, pp. 430435.

[39] K. J. Waldron, J. Estremena, Paul J. Csonka, and S. P. N. Singh, "Analyzing bounding and galloping using simple models," J. Mech. Robot., vol. 1, p. 011002 , Feb. 2008

[40] R. M. Alexander, Elastic Mechanisms in Animal Movement. Great Britain: Cambridge Univ. Press, 1988.

[41] S. Chiaverini, B. Siciliano, and L. Villani, "A survey of robot interaction control schemes with experimental comparison," IEEE/ASME Trans. Mechatronics, vol. 4, no. 3, pp. 273-285, Sep. 1999.

[42] C. Canudas de Wit and R. Kelly, "Passivity analysis of motion controller for robot manipulators with dynamic friction," Asian J. Control, vol. 9, no. 9 , pp. 30-36, 2007.

[43] H. Olsson, K. Astrom, C. Canudas de Wit, M. Gafvert, and P. Lischinky, "Friction models and friction compensation," Eur. J. Control, vol. 4, pp. 176-795, 1998.
[44] P. Armstrong-Helouvry, B. Dupont, and C. Canudas de Wit, "A survey of models, analysis tools and compensation methods for the control of machines with friction," Automatica, vol. 30, no. 7, pp. 1083-1138, 1994.

[45] P. Gonzalez de Santos, E. Garcia, and J. Estremera, Quadrupedal Locomotion: An Introduction to the Control of Four-Legged Robots. London, U.K.: Springer-Verlag, 2006.

[46] U. Schmucker, A. Schneider, and T. Ihme, "Force control in locomotion of legged robots," in Proc. Symp. Robot Control, Nantes, France, 1997, pp. $105-110$.

[47] C. A. Klein and S. Kittivatcharopong, "Optimal distribution for the legs of a walking machine with friction cone constraints," IEEE Trans. Robot. Autom., vol. 6, no. 1, pp. 73-85, Feb. 1990.

[48] J. Or, "A hybrid CPG-ZMP controller for the real-time balance of a simulated flexible spine humanoid robot," IEEE Trans. Syst., Man, Cybern. C, Appl. Rev., vol. 39, no. 5, pp. 547-561, Sep. 2009.

[49] S.-H. Lee and A. Goswami, "Ground reaction force control at each foot: A momentum-based humanoid balance controller for non-level and nonstationary ground," in Proc. IEEE/RSJ Int. Conf. Intell. Robots Syst., Oct., 2010, pp. 3157-3162.

[50] E. Garcia, J. Estremera, and P. Gonzalezde Santos, "A comparative study of stability margins for walking machines," Robot., vol. 20, pp. 595-606, 2002.

[51] B. Son, J. T. Kim, and J. H. Park, "Impedance control for biped robot walking on uneven terrain," in Proc. IEEE Int. Conf. Robot. Biomech., Guilin, China, 2009, pp. 239-244.

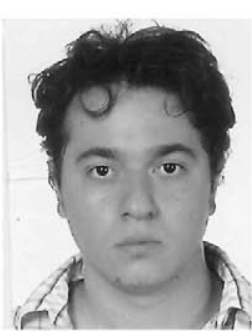

Juan Carlos Arevalo received the B.E degree from the Universidad Simon Bolivar, Miranda State, Venezuela, in 2010 . He is currently working toward the Ph.D. degree at the Universidad Politecnica de Madrid, Madrid, Spain.

He was an Exchange Student with the Royal Institute of Technology (KTH), Stockholm, Sweden, during 2008-2009, and a Visiting Scholar with the Florida Institute for Human and Machine Cognition (IHMC) in 2009. He is carrying out his research activities in the Centre for Automation and Robotics, La Poveda, Spain, where he is working in the HADE project on new actuation systems for achieving high-speed locomotion in walking robots, and the ATLAS project for the development of the technology required to control lower limb exoskeletons, focusing his research in improving the human-machine interaction.

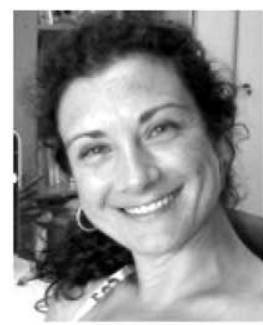

Elena Garcia received the B.E. and Ph.D. degrees from the Universidad Politecnica de Madrid, Madrid, Spain, in 1996 and 2002, respectively.

She is currently a Researcher with the Spanish National Research Council (CSIC), La Poveda, Spain. She joined the Department of Automatic Control of the Institute of Industrial Automation, CSIC, in 1997 and she has been carrying out her research activities in the Centre for Automation and Robotics (CSICUPM) since 2010. She was a Visiting Scholar with the MIT Leg Laboratory in 1998 and with the Laboratoire d'Automatique de Grenoble in 2001. Her research is focused on improving the performance of legged robots, including dynamic stability of legged robots, active compliance in the foot-ground interaction, new actuators for legged robots, agile quadrupeds, and lower limb exoskeletons. She has participated in various research projects in legged locomotion such as ROWER, a walking platform for ship building, SILO4, a four-legged locomotion system used as a test bed in most of her work, and DYLEMA, a project focused on a six-legged walking platform for landmine detection and location. She is currently leading research with the direction of the HADE project on new actuation systems for achieving high-speed locomotion in walking robots, and the ATLAS project for the development of the technology required to control lower limb exoskeletons. She is the coauthor of a scientific book on quadrupedal locomotion, which was published by Springer in 2006 . 